\title{
Performance of Single-tone and Two-tone Frequency-shift Keying for Ultrawideband
}

\author{
Cheng Luo \\ Electrical Engineering \\ and Computer Science, \\ Massachusetts Institute of Tech \\ Cambridge, MA 02139 \\ Email: chengluo@mit.edu
}

\author{
Muriel Médard \\ Electrical Engineering \\ and Computer Science, \\ Massachusetts Institute of Tech \\ Cambridge, MA 02139 \\ Email: medard@mit.edu
}

\begin{abstract}
By using impulsive frequency-shift keying (FSK) with vanishingly small duty cycle, capacity can be achieved in the limit of infinite bandwidth in wideband systems that decorrelate in time and frequency. With limits on bandwidth and peak power, impulsive FSK schemes with small duty cycle can still achieve rates of the order of capacity in ultrawideband systems. We compare performance of single tone and two-tone FSK schemes. Our results indicate that, two-tone FSK can be applicable at lower bandwidth with almost no detriment in the error exponent.
\end{abstract}

\section{Introduction}

The capacity of the infinite-bandwidth general multipath fading channel is equal to the capacity of the infinite-bandwidth additive white Gaussian noise (AWGN) channel with the same average received power constraint. This result has been shown by Kennedy[6], Gallager[5, §8.6] and Telatar and Tse[11]. The proofs are constructive and use frequency-shift keying with non-coherent detection in a system that transmits at a vanishingly low duty cycle. This capacity-achieving transmission scheme, which we term impulsive FSK, is thus "peaky" both in frequency and time. Such a scheme is quite different from the wideband spread-spectrum schemes that create signals that mimic white Gaussian noise (WGN), such as would be optimal for infinite bandwidth nonfading channel. Indeed, the use of signals akin to WGN, which we term bandwidth-scaled, with moments that scale inversely with bandwidth, has been shown to yield a vanishing capacity in the limit of infinite bandwidth ( [11], [8], [10]).

While the results of [6] and [11] only provide a capacity-achieving method for vanishingly small duty cycle and infinite bandwidth, the results do not show how and how fast a system can approach this limit. Recent results show that this limit is approached slowly. In [12], by considering the relation among SNR, capacity and spectral efficiency, Verdú shows that approaching capacity may require extremely large bandwidths and peak-to-average signal ratios. A similar conclusion is reached by Lun, Médard and AbouFaycal in [3], [2] by considering the per codeword probability of error as a function of peak energy, duty cycle and total bandwidth for Rayleigh and general fading channels using impulsive FSK.

From the above discussion emerge two main themes. First, bandwidth-scaled signals, of the type used in commercial direct-sequence code-division multiple access (DS-CDMA) systems, perform poorly in the wideband regime for fading channels. Second, the optimal schemes in the infinite bandwidth regime require extremely high bandwidth and peak power to approach capacity. Both of these results may be viewed as negative results, indicating what certain types of signaling cannot do. What, then, is the appropriate way of transmitting for ultrawideband communications, over bandwidths of the order of several $\mathrm{GHz}$ ? For such bandwidths and moderate signal energy, bandwidth-scaled signals perform poorly. On the other hand, the limitations on bandwidth and peak power in practical systems are well below those required to approach the infinite-bandwidth capacity.

Our goal in this paper is to present a family of signaling schemes which achieve a capacity of the order of the infinite-bandwidth capacity for bandwidths and peak power constraints that are large but well below those required to achieve the infinite bandwidth capacity. The transmission schemes are impulsive single tone FSK and multi-tone FSK with small duty cycle 
and large bandwidth. The multi-tone FSK is introduced in [1] which means several frequencies is used at a time in transmitting a symbol instead of one frequency per symbol scheme. The size of the symbol alphabet is increased while the power per frequency is reduced in order to maintain the overall transmission power constant. We proved that multi-tone FSK can achieve capacity in the infinite bandwidth limit [1]. We study the performance of these schemes in Rayleigh fading channels with limited bandwidth. Our FSK scheme assumes no channel side information at the sender or at the receiver. The multi-tone FSK schemes can be applied at lower bandwidths than traditional single-tone FSK schemes. We also get the error exponents for single-tone and two-tone FSK which are representatives of coding complexity and delay. The two-tone FSK has almost the same performance as single-tone FSK in terms of error exponent.

\section{System model}

The system is studied in Rayleigh fading channel conditions, which are common in wireless communication scenarios. Related work has considered the capacity of FSK schemes. In [9], Stark determined the capacity of FSK schemes under non-selective Rician fading with receiver side information. A similar system is considered in [7] for channels with erasures. However, we consider channels with decorrelation in time and bandwidth and thus none of the above results apply to our model. We send multi-frequency signals which are selected from a large set of frequencies and transmitted using a low duty cycle. We term this signal multi-tone FSK. Because we use a large set of frequencies in a wide bandwidth, the frequency difference between two successive symbols we assume to be greater than the coherence frequency. Moreover, the low duty cycle means successive symbols are generally separated in time by more than a coherence time. Hence, the probability of sending two successive signals within a coherence band in the same coherence time is negligible. We can thus assume different symbols experience independent fading.

We will discuss the model of multi-tone FSK which can be generalized to the FSK case by let the number of tones, $Q$, be 1 . For multi-tone FSK, the channel output $y(t)$ for an input waveform $x(t)$ is given by

$$
y(t)=\sum_{l=1}^{L} a_{l}(t) x\left(t-d_{l}(t)\right)+z(t)
$$

where $L$ is the number of paths, $a_{l}(t)$ and $d_{l}(t)$ are the gain and delay on the $l$ th path at time $t$ respectively, and $z(t)$ is white Gaussian noise with power spectral density $N_{0} / 2$. Let $T_{c}$ and $T_{d}$ be the coherence time and delay spread of the fading channel respectively. We assume that the processes $\left\{a_{l}(t)\right\}$ and $\left\{d_{l}(t)\right\}$ are constant and i.i.d. over time intervals of $T_{c}$ (block-fading model in time), and that $T_{d} \ll T_{c}$ (an underspread channel).

Suppose that the average power constraint is $P$, and let $\theta \in(0,1]$ which is duty cycle. We have $M$ frequencies in which we will send $Q$ of them as Multitone $(Q$-tone) FSK scheme demands. One code word is represented at baseband as the summation of $Q$ complex sinusoids with $Q$ different frequencies of amplitude $\sqrt{P / Q \theta}$, that is

$$
x(t)= \begin{cases}\sqrt{\frac{P}{Q \theta}} \sum_{i \subseteq C_{m}} \exp \left(j 2 \pi f_{i} t\right) & 0 \leq t \leq T_{s}, \\ 0 & \text { otherwise }\end{cases}
$$

where $T_{s}$ satisfies $T_{d}<T_{s} \leq T_{c} . C_{m}$ is a set of $Q$ different integers in $[1, M]$. There are $C_{M}^{Q}$ combinations for the selection of $\mathrm{Q}$ integers, $1 \leq m \leq C_{M}^{Q}$. The frequency $f_{i}$ is chosen such that it is an integer multiple of $1 /\left(T_{s}-T_{d}\right)$.

Let us consider the channel output over the interval $\left[T_{d}, T_{s}\right]$. During this interval, the $\mathrm{Q}$ sinusoids have different channel gains and delays which are constant in the interval owing to the assumptions of the model, and we denote their values by $\left\{a_{l, i}\right\}$ and $\left\{d_{l, i}\right\}$ respectively. Hence by (1), the received signal when a message $m$ is sent is

$$
\begin{aligned}
y(t) & =\sum_{i \subseteq C_{m}} \sum_{l=1}^{L} a_{l, i} \sqrt{\frac{P}{Q \theta}} \exp \left(j 2 \pi f_{i}\left(t-d_{l, i}\right)\right)+z(t) \\
& =\sum_{i \subseteq C_{m}} G_{i} \sqrt{\frac{P}{Q \theta}} \exp \left(j 2 \pi f_{i}\left(t-d_{l, i}\right)\right)+z(t)
\end{aligned}
$$

where $G_{i}=\sum_{l=1}^{L} a_{l, i} \exp \left(-j 2 \pi f_{i} d_{l, i}\right)$ is a complexvalued random variable. We define signal power in the conventional sense as the received signal power, and thus normalize the channel gain so that $E\left[\left|G_{i}\right|^{2}\right]=1$.

At the receiver, we get the correlator outputs

$$
R_{k}=\frac{1}{\sqrt{N_{0}\left(T_{s}-T_{d}\right)}} \int_{T_{d}}^{T_{s}} \exp \left(-j 2 \pi f_{k} t\right) y(t) d t
$$

for $1 \leq k \leq M$. Therefore,

$$
R_{k}= \begin{cases}G_{k} \sqrt{\frac{P\left(T_{s}-T_{d}\right)}{Q \theta N_{0}}}+W_{k} & k \subseteq C_{m}, \\ W_{k} & \text { Otherwise. }\end{cases}
$$

where $\left\{W_{k}\right\}$ is a set of i.i.d. circularly-symmetric complex Gaussian random variables, each satisfying $E\left[\left|W_{k}\right|^{2}\right]=1$. 
Let $\zeta=\frac{P\left(T_{s}-T_{d}\right)}{Q N_{0}}$. When $n \subseteq C_{m}$, the received $\left|R_{n}\right|^{2}$ has the probability density given by (6), otherwise, $\left|R_{n}\right|^{2}$ has the density given by (7).

$$
\begin{gathered}
P_{\left|R_{n}\right|^{2}}(r)=\frac{1}{1+\zeta} \exp \left[\frac{-r}{1+\zeta}\right] \quad(r>0) \\
p_{\left|R_{n}\right|^{2}}(r)=\exp [-r] \quad(r>0) .
\end{gathered}
$$

Keeping the system's average power constant, a change in the duty cycle parameter $\theta$ will affect the signal power $P$.

To decide which signal was transmitted, we use the maximum-a-posteriori (MAP) rule based on the observation of $\left|R_{n}\right|^{2}$ at the receiver. The probability system transmits nothing in a symbol slot is $1-\theta$. When there is no signal being transmitted in a slot, the joint probability density of $\left(\left|R_{1}\right|^{2},\left|R_{2}\right|^{2}, . .,\left|R_{M}\right|^{2}\right)$ is

$$
p\left(r_{1}, r_{2}, \ldots, r_{M}\right)=\prod_{i=1}^{M} \exp \left(-r_{i}\right) .
$$

Otherwise, if one signal is sent, the joint probability density in the slot should be

$p\left(r_{1}, r_{2}, \ldots, r_{M}\right)=\prod_{j \subseteq C_{m}} \frac{\exp \left[\frac{-r_{j}}{1+\zeta}\right]}{1+\zeta} \prod_{i \nsubseteq C_{m}} \exp \left(-r_{i}\right)$.

According to the MAP rule, the summation of the largest $Q$ of $\left|R_{n}\right|^{2}$ s will be compared with a threshold. If $\sum_{\text {largest } Q}\left|R_{n}\right|^{2}$ is greater than $Z$, the receiver will decide that the corresponding signal was transmitted. Otherwise, the decoder declares no signal was transmitted.

Because the symbols experience independent fading, the complex gain of the channel, $A$, decorrelates in any two symbol slots. We compute the capacity of this system using a discrete memoryless channel(DMC) model. The capacity of the system is data rate per symbol over symbol time.

For performance analysis, we restrict ourselves to the 2-tone case. First, we set up a discrete system model. The transmitter sends one of $M(M-1) / 2$ messages which contains two equal power sinusoid waveforms with different frequencies or sends nothing. At the receiver, a bank of matched filters with central frequencies $f_{n}(1 \leq n \leq M)$ are used to detect signals. The detector decodes the message based on $\sum_{\text {largest } 2}\left|R_{n}\right|^{2}$. We select the two largest $\left|R_{n}\right|^{2}$ s, and get their sum. We then compare the sum to a threshold $Z$. The threshold is determined by the MAP rule:

$$
Z=\left(\frac{1+\zeta}{\zeta}\right) \ln \left[(1+\zeta)^{2} \frac{1-\theta}{\theta}\left(\begin{array}{c}
M \\
2
\end{array}\right)\right]
$$

The discrete model associated with this decoding scheme has $M(M-1) / 2+1$ inputs and $M(M-1) / 2+1$ outputs. With probability $\theta$, the transmitter transmits one of $M(M-1) / 2$ messages. Each message has an input probability $\frac{2 \theta}{M(M-1)}$. With probability $1-\theta$, it transmits nothing. The distribution of $\left|R_{n}\right|^{2}$ is known and the decoding rule is determined, so the transition probabilities can be get. The mutual information of the system is optimized on the probability of input, and thus equals capacity for our input alphabet, input distribution constraint and channel transition probabilities.

In section 5, we use numerical methods to calculate the capacity of impulsive FSK and two-tone FSK.

\section{Bounds on capacity}

Before we discuss the capacity of our system, we first consider some bounds on this capacity. From [11], we know that the capacity of the Rayleigh fading channel is the same as that of the AWGN channel in the limit of infinite bandwidth. So a tight bound on capacity is

$$
C=\frac{T_{s}-T_{d}}{T_{s}} F \ln \left(1+\frac{P}{2 N_{0} F}\right)
$$

where $F$ is the bandwidth of the system, $P$ is the average signal power, $N_{0}$ is single-sided power density per dimension for additive noise. The factor $\frac{T_{s}-T_{d}}{T_{s}}$ is introduced because the effective time of transmission is $\left[T_{d}, T_{s}\right]$. When $F$ goes to infinity, the bound approaches $\left(1-\frac{T_{d}}{T_{s}}\right) \frac{P}{2 N_{0}}$. We term this bound the limited energy bound.

Another bound on capacity is deduced from the discrete model of the system. Owing to the limited number of input symbols, an upper bound on FSK capacity is given by

$$
C \leq \frac{\ln (M+1)}{T_{s}} .
$$

This bound is tight when power is very large, as we shall see in following discussion. We denote this bound as the limited bandwidth bound. Similarly, multi-tone FSK has a bound:

$$
C \leq \frac{\ln \left(\left(\begin{array}{c}
M \\
K
\end{array}\right)+1\right)}{T_{s}} .
$$

\section{The error exponents}

The error exponent is very important for studying the relationship between error probability and code length. To discuss the error exponents of this system, we can use the results in [4]:

$$
E_{\text {codebooks }}\left[P_{e, m}\right] \leq 2^{-N\left[E_{0}\left(\rho, P_{X}(x)\right)-\rho R\right]}
$$


where

$$
\begin{aligned}
& E_{0}\left(\rho, P_{X}(x)\right)= \\
& \quad-\log _{2}\left\{\sum_{y}\left[\sum_{x} P_{X}(x) P_{Y \mid X}(y \mid x)^{\frac{1}{1+\rho}}\right]^{1+\rho}\right\}
\end{aligned}
$$

$\rho$ is an arbitrary value between $(0,1) . P_{X}(x)$ is input distribution. $P_{Y \mid X}(y \mid x)$ is the transition probability. Based on our system model, we can have transition probabilities. Let

$$
\operatorname{Er}\left(\rho, R, P_{X}(x)\right)=E_{0}\left(\rho, P_{X}(x)\right)-\rho R
$$

We can get the bound on $E_{\text {codebooks }}\left[P_{e, m}\right]$ :

$$
E_{\text {codebooks }}\left[P_{e, m}\right] \leq 2^{-N E r\left(\rho, R, P_{X}(x)\right)}
$$

Using the Markov inequality, we bound the probability of error as:

$$
P_{e, m} \leq 4 \times 2^{-N E r\left(\rho, R, P_{X}(x)\right)}
$$

Let

$$
\xi(R)=\max _{0 \leq \rho \leq 1} \max _{P_{X}(x)} \operatorname{Er}\left(R, P_{X}(x), \rho\right)
$$

We will evaluate $\xi(R)$ numerically.

\section{Numerical results}

A high peak signal power makes transmission reliable. However, when the average received signal power is constant, we should lower the signal duty cycle in order to improve the peak signal power, which will put a limitation on the data rate. Hence, we need to adjust the duty cycle parameter $\theta$ to optimize the system capacity. In our simulation, all results are optimized with respect to $\theta$ by default.

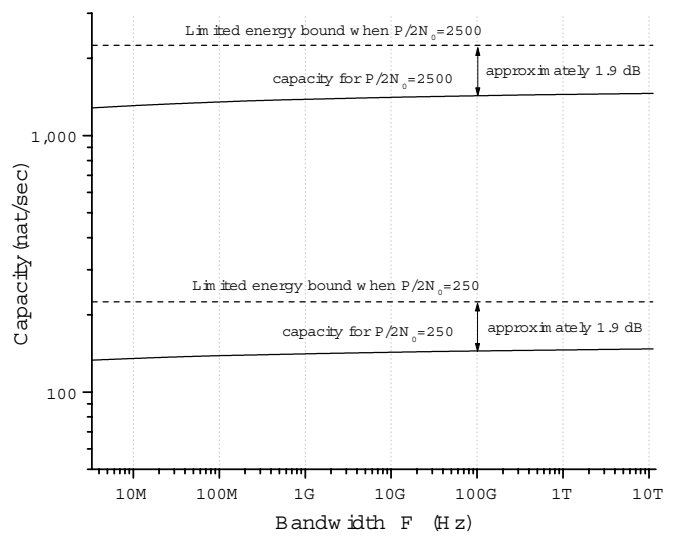

Figure 1: Capacity vs. System Bandwidth. $T_{s}=$ $10 \mu s, T_{d}=1 \mu s$.

In Figure 1, we fix power, and find, as expected, that the capacity of FSK increases with the bandwidth of system. However, it grows very slowly, and has roughly a gap of $2 \mathrm{~dB}$ with the infinite bandwidth bound when the system bandwidth is between $1 \mathrm{MHz}$ and $10 T H z$. Note that our scheme achieves at moderate bandwidths capacities very close to those achievable under very large bandwidths.

For bandwidth $F=1 M H z$, we compare the capacity of 2-tone FSK system and that of FSK system in different power conditions. The simulation results are shown in Figure 2. The capacity of 2-tone FSK system is a little bit lower than that of the FSK system where the limited energy bound is a main limitation for the FSK system. When power is very large, the limited bandwidth bound will limit the system performance. In this region, the capacity of 2-tone FSK will exceed that of FSK, because Multi-tone FSK has a higher limited bandwidth bound. We can also see that for moderate received power, the limitation in bandwidth does not hamper these schemes, which achieve capacities of the order of the limited energy bound.

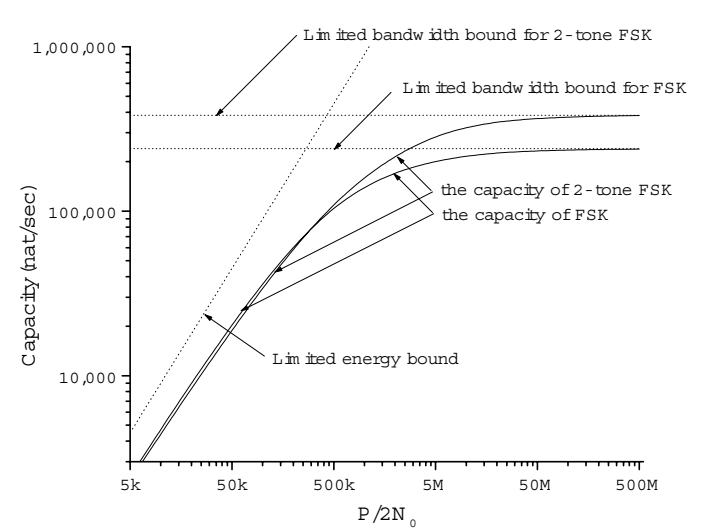

Figure 2: FSK vs. 2-tone FSK with fixed bandwidth $F=1 M H z, T_{d}=1 \mu s, T_{s}=10 \mu s$.

The limited bandwidth bound will increase with $M$ while the limited energy bound is not changed with bandwidth. Figure 3 shows how the capacities of 2tone FSK and FSK are changed with $M$. We use $T_{s}=$ $0.1 s$ and $\frac{P}{2 N_{0}}=40$ here. $M$ is related to bandwidth by $F=M /\left(T_{s}-T_{d}\right)$.

While multi-tone FSK does better in some regions, its error exponents may be a little worse than that of FSK, which means it needs a longer code length to achieve the same error probability. Figure 4 shows the error exponents of FSK and multi-tone FSK under the same power and bandwidth condition. The capacity of multi-tone FSK is higher in this case, when data rate is low, FSK need shorter code length for achieving the same error probability as multi-tone FSK. However, the performances of the two schemes are quite close. 


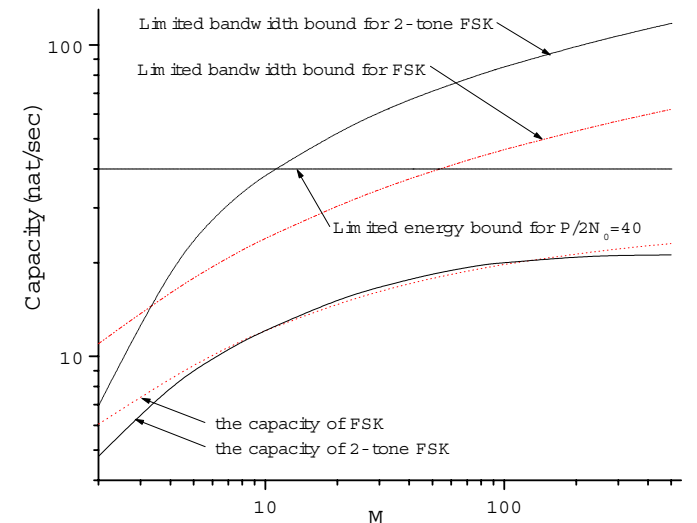

Figure 3: FSK vs. 2-tone FSK with fixed average power, $\frac{P}{2 N_{0}}=40, T_{s}=0.1 s, T_{d}=1 \mu s$.

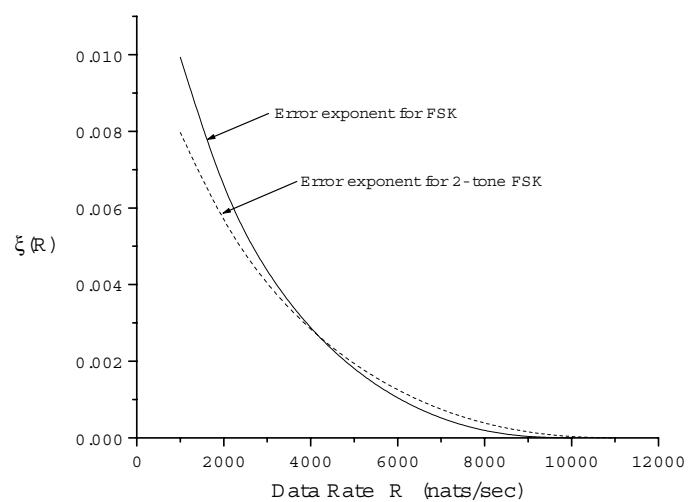

Figure 4: Error exponents for FSK and 2-tone FSK with $F=5 \mathrm{MHz}, T_{d}=1 \mu \mathrm{s}, T_{s}=10 \mu \mathrm{s}, \theta=0.01$, $P / 2 N_{0}=50000$.

\section{Conclusions}

The capacity grows slowly with bandwidth, and is nearly $2 \mathrm{~dB}$ lower than the infinite bandwidth bound for bandwidths commensurate with general mobile communication conditions. Using a moderate bandwidth, we can approach the capacity achieved by using very large bandwidth. Multi-tone FSK can get a higher capacity than FSK when bandwidth is limited and power can be sufficiently large. The error exponent will almost not be hurt when the number of tones is small.

\section{References}

[1] C. Luo, M. Médard. Frequency-shift keying for ultrawideband - achieving rates of the order of capacity. In 40th Annual Allerton Conference on Communication, Control, and Computing, October 2002 .
[2] D. S. Lun, M. Médard and I. C. Abou-Faycal. Error exponents for capacity-achieving signaling on wideband rayleigh fading channels. In International Symposium on Information Theory and its Applications, October 2002.

[3] D. S. Lun, M. Médard and I. C. Abou-Faycal. Error exponents for wideband multipath fading channels - a strong coding theorem. In Proc. of Conference on Information Sciences and Systems (CISS), March 2002.

[4] Robert G. Gallager. A simple derivation of the coding theorem and some applications. IEEE Trans. Inform. Theory, pages 3-18, January 1965.

[5] Robert. G. Gallager. Information Theory and Reliable Communication. John Wiley \& Sons, New York, NY, 1968.

[6] Robert .S. Kennedy. Fading Dispersive Communication Channels. Wiley Interscience, New York, NY, 1969.

[7] A. Matache and J.A. Ritcey. Optimum code rates for noncoherent mfsk with errors and erasures decoding over rayleigh fading channels. In Proc. of of the Thirty-First Asilomar Conference on Signals, Systems and Computers, pages 62-66, 1997.

[8] M. Médard and R.G. Gallager. Bandwidth scaling for fading multipath channels. IEEE Transactions on Information Theory, 48:840-852, 2002.

[9] W.E. Stark. Capacity and cutoff rate of noncoherent fsk with nonselective rician fading. IEEE Transactions on Communications, COM33(11):1153-1159, November 1985.

[10] V.G. Subramanian and B. Hajek. Broad-band fading channels: signal burstiness and capacity. IEEE Transactions on Information Theory, 48(4):809-827, 2002.

[11] I. Emre Telatar and David N.C. Tse. Capacity and mutual information of wideband multipath fading channels. IEEE Trans. Inform. Theory, 46(4):1384-1400, July 2000.

[12] S. Verdú. Spectral efficiency in the wideband regime. IEEE Trans. Inform. Theory, 48(6):1319 -1343 , June 2002. 